\title{
The effect of axial force on the free vibration of an Euler-Bernoulli beam carrying a number of various concentrated elements
}

\author{
Gürkan Şakar \\ Department of Mechanical Engineering, Atatürk University, 25240 Erzurum, Turkey \\ E-mail: gsakar@atauni.edu.tr
}

Received 19 June 2012

Revised 4 October 2012

Accepted 19 October 2012

\begin{abstract}
In this study, free vibration analysis of beams carrying a number of various concentrated elements including point masses, rotary inertias, linear springs, rotational springs and spring-mass systems subjected to the axial load was performed. All analyses were performed using an Euler beam assumption and the Finite Element Method. The beam used in the analyses is accepted as pinned-pinned. The axial load applied to the beam from the free ends is either compressive or tensile. The effects of parameters such as the number of spring-mass systems on the beam, their locations and the axial load on the natural frequencies were investigated. The mode shapes of beams under axial load were also obtained.
\end{abstract}

Keywords: Concentrated elements, natural frequency, finite element method, axial load

\section{Introduction}

The use of beams as structural components in civil, aeronautical and mechanical engineering is a commom practice. The flexural vibration of beams can be affected by many factors such as the axial load, intermediate supports, attached masses and springs. Determination of the influence of these parameters on the vibration of beams is of practical interest in numerous engineering applications. Hence, a great deal of work has been carried out on the natural frequencies and corresponding mode shapes of beams under axial loading.

A summary of the natural frequencies of beams subjected to axial load has been given by Blevins [1], Weaver et al. [2]. Borbon and Ambrosini [3] presented a numerical-experimental study on natural frequencies of thin-walled beams axially loaded. Chen and $\mathrm{Wu}$ [4] obtained the natural frequencies and corresponding mode shapes of nonuniform beams with multiple spring-mass systems using the numerical assembly method. Chen [5], Lin and Tsai [6] employed the Numerical Assembly Method (NAM) to determine the exact values of natural frequencies and associated mode shapes of a multi-span beam carrying a number of masses, spring-mass systems. Gürgöze [7] studied the natural vibration problem of a mechanical system consisting of a clamped free Bernoulli-Euler beam to which several spring-mass-systems are attached in span. Hashemi and Richard [8] presented a dynamic finite element for the natural frequencies and modes calculation of coupled bending-torsional vibration of axially loaded beams based on the closed form solutions of the Bernoulli-Euler and St. Venant beam theories. Lin and Tsai [9] also investigated the natural frequencies and associated mode shapes of multi-step beam carrying a number of point masses and rotary inertias. Lin and Tsai also [10] determined the "exact" solutions for the natural frequencies and mode shapes of a uniform multi-span beam carrying multiple spring-mass systems. Lin [11] determined the natural frequencies and mode shapes of multi-span Timoshenko beams carrying a number of various concentrated elements including point 
masses, rotary inertias, linear springs, rotational springs and spring-mass systems subjected to a harmonic force using the numerical assembly method. Lin [12] determined the "exact" frequency response amplitudes of a multispan beam carrying a number of various concentrated elements and subjected to a harmonic force, and the exact natural frequencies and mode shapes of the beam for the zero harmonic force using the numerical assembly method. Naguleswaran [13] obtained the natural frequency values of the beams on up to five resilient supports including ends and carrying several particles using the Bernoulli-Euler Beam Theory and fourth-order determinant equated to zero. Naguleswaran [14] found the natural frequencies of an Euler Bernoulli beam with up to five elastic supports (including the ends of the beam) by setting a fourth-order determinant to zero. Wang et al. [15] studied the natural frequencies and mode shapes of a uniform Timoshenko beam carrying multiple intermediate spring-mass systems with the effects of shear deformation and rotary inertia. Wu and Chou [16] presented a numerical technique to obtain the exact solutions for the lowest several natural frequencies and mode shapes of a uniform beam carrying any number of spring-mass systems with various boundary conditions. Wu and Chen [17] presented a modified lumped-mass transfer matrix method for the free vibration analysis of a multistep Timoshenko beam carrying eccentric lumped masses with eccentricity and rotary inertias. Yesilce and Demirdag [18] determined the exact solutions for the first five natural frequencies and mode shapes of a Timoshenko multi-span subjected to the axial force.

Although numerous studies have been performed in the area of free vibration analysis of Euler-Bernoulli singlespan beams carrying a number of spring-mass systems, Euler-Bernoulli multi-span beams carrying a number of spring-mass systems and Euler-Bernoulli single and multiple-span beams carrying a number of various concentrated elements, where only a few studies have been performed on free vibration analysis of Euler-Bernoulli multiple-span beams carrying a number of various concentrated elements subjected to axial load (compressive). According to author's knowledge there are no other studies on natural ferquencies and mode shapes of Euler-Bernoulli multispan beams carrying a number of concentrated elements (point masses, rotary inertias, linear springs, rotational springs, mass-spring systems) with axial load (comporessive and tensile). This paper aims to determine the natural frequencies and mode shapes of Euler-Bernoulli multiple-span beams carrying a number of various concentrated elements with axial load effect.

\section{Theoretical analysis}

The beam used in the study is shown in Fig. 1. It carries three point masses with rotary inertias at three locations shown as 1, 6, 8, two linear springs, two rotational springs at the other two locations shown as 2,4 , and two springmass systems shown as 5, 9. The beam is subjected to axial force as compressive or tensile. The beam is assumed to be an Euler beam and the finite element model is developed to represent the variation of a uniform cross-section. As shown in Fig. 2 an elemental finite element has four degrees of freedom. They are called nodal displacements $\left(w_{1}, w_{2}\right)$ and slopes $\left(\theta_{1}, \theta_{2}\right)$.

It assumed that the transverse displacement variation in the $\mathrm{x}$ direction along the beam length is a cubic function in $x$ :

$$
w(x)=C_{1}+C_{2} x+C_{3} x^{2}+C_{4} x^{3}
$$

where the constants $C_{1}, C_{2}, C_{3}$ and $C_{4}$ are in general, functions of time and can be determined from the boundry conditions. The boundry conditions in general form, are

$$
\begin{array}{ll}
x=0 \Rightarrow & w(0)=w_{1} \\
x=0 \Rightarrow & \frac{\partial w(0)}{\partial x}=\theta_{1} \\
x=\ell \Rightarrow & w(\ell)=w_{2} \\
x=\ell \Rightarrow & \frac{\partial w(\ell)}{\partial x}=\theta_{2}
\end{array}
$$


Differentiating Eq. (1) with respect to $x$ and substituting the boundry conditions, Eqs (2)-(5) into Eq. (1) and its derivative the four constants $C_{1}$ to $C_{4}$ can be found. Substituting these constans into Eq. (1) and rearranging the terms so that each degree of freedom is in separate term, the following expression is obtained;

$$
w(x)=\left(1-\frac{3 x^{2}}{\ell^{2}}+\frac{2 x^{3}}{\ell^{3}}\right) w_{1}+\left(x-\frac{2 x^{2}}{\ell}+\frac{x^{3}}{\ell^{2}}\right) \theta_{1}+\left(\frac{3 x^{2}}{\ell^{2}}-\frac{2 x^{3}}{\ell^{3}}\right) w_{2}+\left(-\frac{x^{2}}{\ell}+\frac{x^{3}}{\ell^{2}}\right) \theta_{2}
$$

The strain energy $U$ of an elemental length $\ell$ of an Euler beam is given by;

$$
U=\frac{1}{2} \int_{0}^{\ell} E I\left(\frac{d^{2} w(x)}{d x^{2}}\right)^{2} d x
$$

Differentiating Eq. (6) twice with rerspect to $x$, substituting into Eq. (7), carrying out the integration and writing in matrix form yields,

$$
U=\frac{1}{2}\{q\}^{T}\left[k_{e}\right]\{q\}
$$

Nodal coordinate vector

$$
\{q\}^{T}=\left\{\begin{array}{llll}
w_{1} & \theta_{1} & w_{2} & \theta_{2}
\end{array}\right\}
$$

The $V$ denotes the work carried out by an axial force;

$$
V=\frac{1}{2} F \int_{0}^{\ell}\left(\frac{d w(x)}{d x}\right)^{2} d x
$$

Differentiating Eq. (6) with rerspect to $x$, substituting into Eq. (10), carrying out the integration and writing in matrix form yields,

$$
V=\frac{1}{2}\{q\}^{T}\left[k_{g e}\right]\{q\}
$$

The kinetic energy $T$ of an elemental length $\ell$ of an Euler beam is given by;

$$
T=\frac{1}{2} \int_{0}^{\ell} \rho A\left(\frac{d w(x)}{d t}\right)^{2} d x
$$

Differentiating Eq. (6) with rerspect to time, substituting into Eq. (12), carrying out the integration and writing in matrix form yields,

$$
T=\frac{1}{2}\{\dot{q}\}^{T}\left[m_{e}\right]\{\dot{q}\}
$$

After applying the standard procedure for a finite element analysis for a beam element elastic stiffness matrix $\left[k_{e}\right]$, element geometrical stiffness matrix $\left[k_{g e}\right]$ and element mass matrix $\left[m_{e}\right]$ are obtained respectively.

In the study global elastic stiffness, geometrical stiffness and mass matrices are formed using the individual stiffness, geometrical stiffness and mass matrices of each beam element. The diagonal stiffness matrices of concentrated elements (linear and torsional springs, spring-mass systems) and mass matrices of concentrated elements (point masses, rotary inertias, spring-mass systems) are then placed into the diagonal location of global elastic stiffness and the global mass matrix respectively. The dynamic response of a beam carrying a number of concentrated elements can be formulated by means of Lagrange's equation of motion in which the external forces are expressed in terms of potentials. Performing the required operations to the entire system leads to the governing matrix equation of

$$
\left[M_{e}\right]\{\ddot{q}\}+\left[\left[K_{e}\right]-F\left[K_{g e}\right]\right]\{q\}=0
$$

where the matrices $\left[K_{e}\right],\left[K_{g e}\right]$ and $\left[M_{e}\right]$ are global elastic stiffness, global geometrical stiffness and global mass matrices, respectively. The natural frequencies of an Euler-Bernoulli beam subjected to axial load (compressive (-) or tensile $(+))$ are obtained from the following equations.

$$
\begin{array}{ll}
{\left[\left[\left[K_{e}\right]-F\left[K_{g e}\right]\right]-\omega^{2}\left[M_{e}\right]\right]\{q\}=0} & \text { (For compressive loads) } \\
{\left[\left[\left[K_{e}\right]+F\left[K_{g e}\right]\right]-\omega^{2}\left[M_{e}\right]\right]\{q\}=0} & \text { (For tensile loads) }
\end{array}
$$


Table 1

Comparison of natural frequencies of a uniform beam used in example 1

\begin{tabular}{lccccc}
\hline Boundary condition & Methods & \multicolumn{4}{c}{ Natural frequencies (rad/s) } \\
\cline { 3 - 6 } & & $\omega_{1}$ & $\omega_{2}$ & $\omega_{3}$ & $\omega_{4}$ \\
\hline Pinned-pinned & Present & 2809.175 & 4333.995 & 5479.463 & 8504.874 \\
& Ref. [10] & 2808.816 & 4333.442 & 5478.764 & 8503.790 \\
\hline
\end{tabular}

Table 2

The lowest five natural frequencies of the beam used in example 2

\begin{tabular}{|c|c|c|c|c|c|c|c|c|}
\hline \multirow{2}{*}{$\begin{array}{l}\text { Number } \\
\text { of span }\end{array}$} & \multirow[t]{2}{*}{ Mode } & \multicolumn{7}{|c|}{ Natural frequencies $(\mathrm{rad} / \mathrm{s})$} \\
\hline & & $F_{r}=0$ & $F_{r}=-0.25$ & $F_{r}=-0.5$ & $F_{r}=-0.75$ & $F_{r}=0.25$ & $F_{r}=0.5$ & $F_{r}=0.75$ \\
\hline \multirow[t]{5}{*}{1} & 1 & 499.908 & 465.544 & 428.307 & 387.348 & 531.970 & 562.135 & 590.702 \\
\hline & 2 & 1665.167 & 1618.490 & 1570.323 & 1520.527 & 1710.478 & 1754.527 & 1797.408 \\
\hline & 3 & 3105.025 & 3064.743 & 3023.908 & 2982.499 & 3144.776 & 3184.015 & 3222.762 \\
\hline & 4 & 5396.642 & 5362.618 & 5328.137 & 5293.185 & 5430.218 & 5463.359 & 5496.074 \\
\hline & 5 & 7597.157 & 7562.992 & 7528.593 & 7493.961 & 7631.090 & 7664.788 & 7698.253 \\
\hline \multirow[t]{5}{*}{2} & 1 & 860.351 & 831.609 & 801.816 & 770.848 & 888.144 & 915.074 & 941.216 \\
\hline & 2 & 2815.244 & 2780.768 & 2745.777 & 2710.248 & 2849.228 & 2882.737 & 2915.792 \\
\hline & 3 & 5033.503 & 4993.412 & 4952.921 & 4912.021 & 5073.200 & 5112.512 & 5151.444 \\
\hline & 4 & 6023.615 & 5998.758 & 5973.736 & 5948.548 & 6048.314 & 6072.858 & 6097.252 \\
\hline & 5 & 8781.495 & 8763.059 & 8744.501 & 8725.817 & 8799.809 & 8818.006 & 8836.085 \\
\hline \multirow[t]{5}{*}{3} & 1 & 2809.175 & 2774.859 & 2740.028 & 2704.66 & 2842.998 & 2876.347 & 2909.241 \\
\hline & 2 & 4333.995 & 4303.837 & 4273.243 & 4242.201 & 4363.732 & 4393.060 & 4421.992 \\
\hline & 3 & 5479.463 & 5446.682 & 5413.824 & 5380.892 & 5512.166 & 5544.789 & 5577.329 \\
\hline & 4 & 8504.874 & 8490.039 & 8475.098 & 8460.047 & 8519.603 & 8534.228 & 8548.752 \\
\hline & 5 & 11454.391 & 11438.941 & 11423.319 & 11407.520 & 11469.674 & 11484.794 & 11499.757 \\
\hline
\end{tabular}

\section{Numerical results and discussion}

The free vibration analysis of an axially loaded uniform Euler-Bernoulli beam carrying various concentrated elements is investigated using FEM. The dimensions and physical properties for the uniform beam have been taken from reference [11]. These parameters are given as follows; Young's modulus $E=2.069 \times 10^{11} \mathrm{~N} / \mathrm{m}^{2}$, diameter $d=0.05 \mathrm{~m}$, moment of inertia of cross-sectional area $I=3.06796 \times 10^{-7} \mathrm{~m}^{4}$, mass per unit length $\bar{m}=$ $15.3879 \mathrm{~kg} / \mathrm{m}$, and total length $L=1 \mathrm{~m}$, total mass $m_{b}=\bar{m} L=15.3875 \mathrm{~kg}$, reference spring constant $k_{b}=$ $E I / L^{3}=6.34761 \times 10^{4} \mathrm{~N} / \mathrm{m}$. Different from reference [11] for the spring-mass effect $\left(k_{e}^{*}=k_{e} L^{3} /(E I), m_{e}^{*}=\right.$ $\left.m_{e} /(\bar{m} L)\right)$ and for the axial force effect $F_{r}=\frac{F L^{2}}{\pi^{2} E I}=0,0.25,0.5,0.75$ are introduced. In FEM, the two-node beam elements are used and each continuous beam is subdivided into 80 beam elements.

The beam compared in example 1 is shown in Fig. 3. It is a uniform pinned-pinned beam carrying three point masses with rotary inertias at three locations, two linear springs and two rotational springs at the other two locations and two intermediate pinned supports without an external load. The given data for the three point masses and three rotary inertias are: $m_{1}^{*}=m_{1} /(\bar{m} L)=0.3, m_{5}^{*}=0.5, m_{7}^{*}=0.9, J_{1}^{*}=J_{1} /\left(\bar{m} L^{3}\right)=0.001, J_{5}^{*}=0.002, J_{7}^{*}=$ $0.003, \xi_{1}=x_{1} / L=0.1, \xi_{5}=0.6$ and $\xi_{7}=0.8$, respectively; those for the two linear springs and two rotational springs are; $K_{T 2}^{*}=K_{T 2} L^{3} /(E I)=10, K_{T 4}^{*}=20, K_{R 2}^{*}=K_{R 2} L /(E I)=3, K_{R 4}^{*}=4$ located at $\xi_{2}=0.2$, and $\xi_{4}=0.4$ respectively, and the two intermediate pinned supports are located at $\xi_{3}=0.3$, and $\xi_{6}=0.7$.

To validate the FEM method the example studied by Lin, [11] was used and the corresponding results were obtained. Table 1 shows a comparison with the numerical results of Lin, [11] based on NAM (Numerical Assembly Method), with present results obtained using FEM. Note that since the results of reference [11], are given as dimensionless frequency parameters they are all converted to the frequencies in $\mathrm{rad} / \mathrm{s}$ in Table 1. It can be observed that the present results are in agreement with that of Lin, [11]. In order to study the influence of an axial load in the natural frequencies of a multi-span uniform beam carrying a number of various concentrated elements, the physical model shown in Fig.1 is used. All the calculations are performed as compression force (-) and tensile force (+) in the study, and they are denoted as first case and second case in the corresponding tables, respectively.

The beam studied in example 2 is shown in Fig. 3. It is a uniform pinned-pinned beam carrying three point masses with rotary inertias at three locations, two linear springs and two rotational springs at the other two locations. For this 
Table 3

The lowest five natural frequencies of the single-span beam used in examle 3

\begin{tabular}{|c|c|c|c|c|c|c|c|c|}
\hline \multirow{2}{*}{$\begin{array}{l}\text { Number of spring- } \\
\text { mass system }\end{array}$} & \multirow[t]{2}{*}{ Mode } & \multicolumn{7}{|c|}{ Natural frequencies (rad/s) } \\
\hline & & $F_{r}=0$ & $F_{r}=-0.25$ & $F_{r}=-0.5$ & $F_{r}=-0.75$ & $F_{r}=0.25$ & $F_{r}=0.5$ & $F_{r}=0.75$ \\
\hline \multirow[t]{5}{*}{0} & 1 & 499.908 & 465.544 & 428.307 & 387.348 & 531.970 & 562.135 & 590.702 \\
\hline & 2 & 1665.167 & 1618.490 & 1570.323 & 1520.527 & 1710.478 & 1754.527 & 1797.408 \\
\hline & 3 & 3105.025 & 3064.743 & 3023.908 & 2982.499 & 3144.776 & 3184.015 & 3222.762 \\
\hline & 4 & 5396.642 & 5362.618 & 5328.137 & 5293.185 & 5430.218 & 5463.359 & 5496.074 \\
\hline & 5 & 7597.157 & 7562.992 & 7528.593 & 7493.961 & 7631.090 & 7664.788 & 7698.253 \\
\hline \multirow[t]{5}{*}{1} & 1 & 409.796 & 389.906 & 365.729 & 336.445 & 426.068 & 439.323 & 450.107 \\
\hline & 2 & 633.986 & 620.277 & 608.123 & 597.545 & 649.117 & 665.449 & 682.720 \\
\hline & 3 & 1676.140 & 1629.684 & 1581.758 & 1532.224 & 1721.244 & 1765.100 & 1807.800 \\
\hline & 4 & 3108.837 & 3068.686 & 3027.989 & 2986.724 & 3148.464 & 3187.586 & 3226.220 \\
\hline & 5 & 5400.686 & 5366.703 & 5332.261 & 5297.349 & 5434.223 & 5467.325 & 5500.001 \\
\hline \multirow[t]{5}{*}{2} & 1 & 283.346 & 282.470 & 280.974 & 277.894 & 283.923 & 284.333 & 284.640 \\
\hline & 2 & 413.016 & 394.236 & 371.819 & 345.898 & 428.517 & 441.211 & 451.580 \\
\hline & 3 & 636.275 & 622.385 & 610.029 & 599.247 & 651.555 & 667.999 & 685.343 \\
\hline & 4 & 1678.276 & 1631.876 & 1584.011 & 1534.545 & 1723.330 & 1767.140 & 1809.799 \\
\hline & 5 & 3110.282 & 3070.159 & 3029.491 & 2988.257 & 3149.882 & 3188.978 & 3227.587 \\
\hline
\end{tabular}

Table 4

The lowest five natural frequencies of the two-span beam used in example 4. $\left(\xi_{3}=0.3\right)$

\begin{tabular}{|c|c|c|c|c|c|c|c|c|}
\hline \multirow{2}{*}{$\begin{array}{l}\text { Number of spring- } \\
\text { mass system }\end{array}$} & \multirow[t]{2}{*}{ Mode } & \multicolumn{7}{|c|}{ Natural frequencies (rad/s) } \\
\hline & & $F_{r}=0$ & $F_{r}=-0.25$ & $F_{r}=-0.5$ & $F_{r}=-0.75$ & $F_{r}=0.25$ & $F_{r}=0.5$ & $F_{r}=0.75$ \\
\hline \multirow[t]{5}{*}{0} & 1 & 860.351 & 831.609 & 801.816 & 770.848 & 888.144 & 915.074 & 941.216 \\
\hline & 2 & 2815.244 & 2780.768 & 2745.777 & 2710.248 & 2849.228 & 2882.737 & 2915.792 \\
\hline & 3 & 5033.503 & 4993.412 & 4952.921 & 4912.021 & 5073.200 & 5112.512 & 5151.444 \\
\hline & 4 & 6023.615 & 5998.758 & 5973.736 & 5948.548 & 6048.314 & 6072.858 & 6097.252 \\
\hline & 5 & 8781.495 & 8763.059 & 8744.501 & 8725.817 & 8799.809 & 8818.006 & 8836.085 \\
\hline \multirow[t]{5}{*}{1} & 1 & 511.843 & 510.760 & 509.438 & 507.786 & 512.746 & 513.513 & 514.172 \\
\hline & 2 & 876.735 & 849.128 & 820.715 & 791.465 & 903.576 & 929.698 & 955.1426 \\
\hline & 3 & 2826.761 & 2792.484 & 2757.702 & 2722.392 & 2860.553 & 2893.879 & 2926.757 \\
\hline & 4 & 5034.429 & 4994.330 & 4953.831 & 4912.923 & 5074.134 & 5113.453 & 5152.393 \\
\hline & 5 & 6026.380 & 6001.565 & 5976.586 & 5951.441 & 6051.038 & 6075.540 & 6099.893 \\
\hline \multirow[t]{5}{*}{2} & 1 & 285.356 & 285.225 & 285.071 & 284.889 & 285.471 & 285.571 & 285.659 \\
\hline & 2 & 511.990 & 510.943 & 509.671 & 508.090 & 512.867 & 513.613 & 514.256 \\
\hline & 3 & 881.140 & 853.707 & 825.483 & 796.438 & 907.823 & 933.799 & 959.110 \\
\hline & 4 & 2829.057 & 2794.807 & 2760.053 & 2724.773 & 2862.822 & 2896.123 & 2928.976 \\
\hline & 5 & 5034.879 & 4994.785 & 4954.290 & 4913.387 & 5074.580 & 5113.896 & 5152.832 \\
\hline
\end{tabular}

example, the used data for the beam and concentrated elements are the same as in example 1. The load was applied from the two ends as compressive (-) or tensile $(+)$ force. In this example, the effects of a number of spans on the natural frequencies were examined. Firstly, single span, then two span $\left(\xi_{3}=0.3\right)$ and finally three span $\left(\xi_{3}=0.3\right.$ and $\left.\xi_{6}=0.7\right)$ beams were analysed. It can be seen from Table 2 that when the number of spans increases all natural frequencies increase in both compressive and tensile load conditions. Note that a compressive axial force reduces the natural frequencies whereas a tensile force increases them as expected. The effect of compressive axial force is predominant over those of the corresponding tensile force on the natural frequencies of a uniform beam supported by pins, carrying various concentrated elements. From Table 2 it can also be seen that compressive and tensile forces are significant in the lower frequencies. However the effect of both load conditions has little influence on the higher natural frequencies.

Figures 4, 5 and 6 show the mode shapes of uniform single, two and three-span beams, respectively, carrying various concentrated elements subjected to compressive axial load. For each case the $F_{r}$ value was taken as 0.5 .

The beam studied in example 3 is presented in Fig. 3. The data is the same as that of example 2. Additionally, two spring-mass systems are added to the beam. The given data for the two spring-mass systems are; $k_{e 5}^{*}=k_{e 5} L^{3} /(E I)=20, k_{e 9}^{*}=10, \xi_{5}=0.5, \xi_{9}=0.9, m_{e 5}^{*}=m_{e 5} /(\bar{m} L)=0.3, m_{e 5}^{*}=0.5, \xi_{5}=0.5$, and $\xi_{9}=0.9$, respectively. In this example the effect of spring-mass number is investigated. The frequency 
Table 5

The lowest five natural frequencies of the three-span beam used in example 5. $\left(\xi_{3}=0.3, \xi_{7}=0.7\right)$

\begin{tabular}{|c|c|c|c|c|c|c|c|c|}
\hline \multirow{2}{*}{$\begin{array}{l}\text { Number of spring- } \\
\text { mass system }\end{array}$} & \multirow[t]{2}{*}{ Mode } & \multicolumn{7}{|c|}{ Natural frequencies $(\mathrm{rad} / \mathrm{s})$} \\
\hline & & $F_{r}=0$ & $F_{r}=-0.25$ & $F_{r}=-0.5$ & $F_{r}=-0.75$ & $F_{r}=0.25$ & $F_{r}=0.5$ & $F_{r}=0.75$ \\
\hline \multirow[t]{5}{*}{0} & 1 & 2809.175 & 2774.859 & 2740.028 & 2704.66 & 2842.998 & 2876.347 & 2909.241 \\
\hline & 2 & 4333.995 & 4303.837 & 4273.243 & 4242.201 & 4363.732 & 4393.060 & 4421.992 \\
\hline & 3 & 5479.463 & 5446.682 & 5413.824 & 5380.892 & 5512.166 & 5544.789 & 5577.329 \\
\hline & 4 & 8504.874 & 8490.039 & 8475.098 & 8460.047 & 8519.603 & 8534.228 & 8548.752 \\
\hline & 5 & 11454.391 & 11438.941 & 11423.319 & 11407.520 & 11469.674 & 11484.794 & 11499.757 \\
\hline \multirow[t]{5}{*}{1} & 1 & 521.262 & 521.190 & 521.114 & 521.034 & 521.330 & 521.396 & 521.458 \\
\hline & 2 & 2819.294 & 2785.176 & 2750.553 & 2715.402 & 2852.927 & 2886.094 & 2918.814 \\
\hline & 3 & 4340.811 & 4310.637 & 4280.027 & 4248.966 & 4370.560 & 4399.899 & 4428.840 \\
\hline & 4 & 5480.688 & 5447.963 & 5415.164 & 5382.294 & 5513.337 & 5545.907 & 5578.398 \\
\hline & 5 & 8505.892 & 8491.070 & 8476.140 & 8461.102 & 8520.609 & 8535.223 & 8549.735 \\
\hline \multirow[t]{5}{*}{2} & 1 & 286.762 & 286.753 & 286.743 & 286.733 & 286.771 & 286.780 & 286.788 \\
\hline & 2 & 521.263 & 521.191 & 521.116 & 521.036 & 521.332 & 521.397 & 521.459 \\
\hline & 3 & 2821.975 & 2787.885 & 2753.290 & 2718.169 & 2855.580 & 2888.721 & 2921.415 \\
\hline & 4 & 4342.686 & 4312.530 & 4281.938 & 4250.895 & 4372.419 & 4401.741 & 4430.665 \\
\hline & 5 & 5480.910 & 5448.195 & 5415.407 & 5382.548 & 5513.549 & 5546.110 & 5578.592 \\
\hline
\end{tabular}

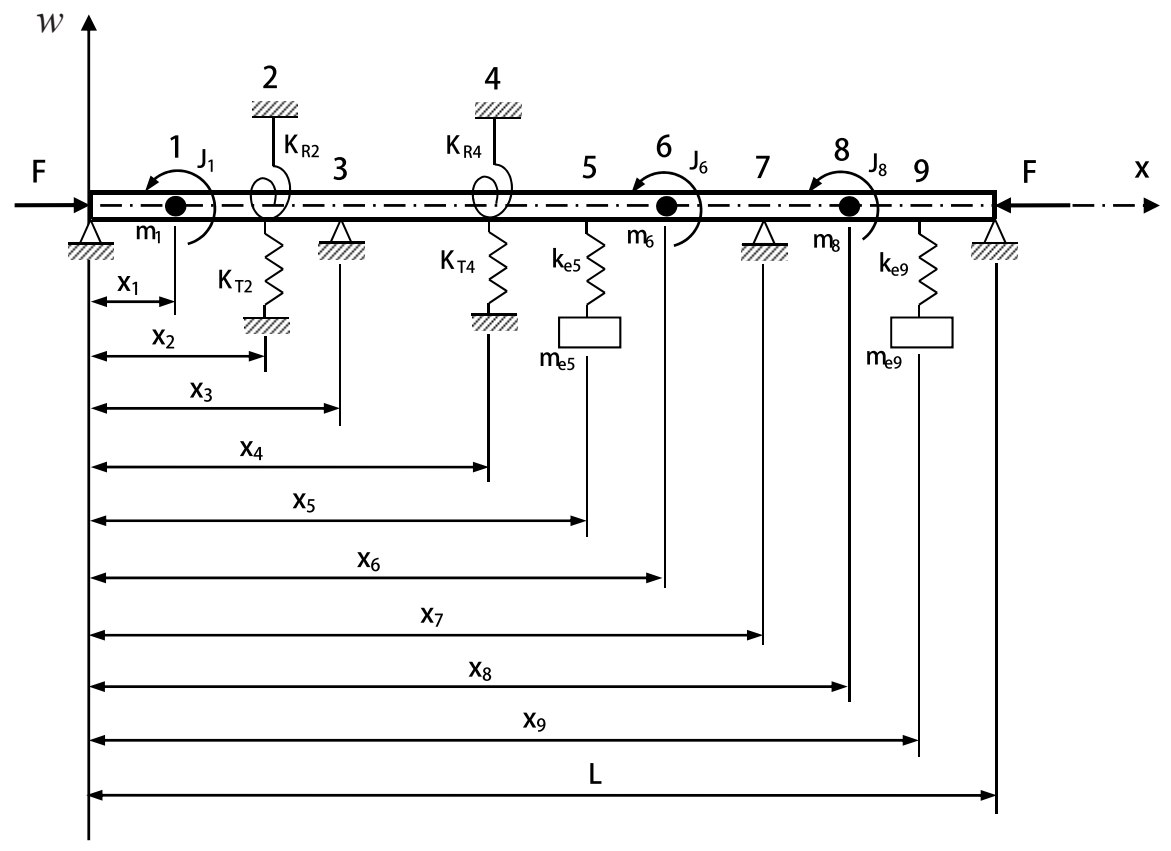

Fig. 1. A uniform beam supported by pins, carrying various concentrated elements and subjected to axial load.

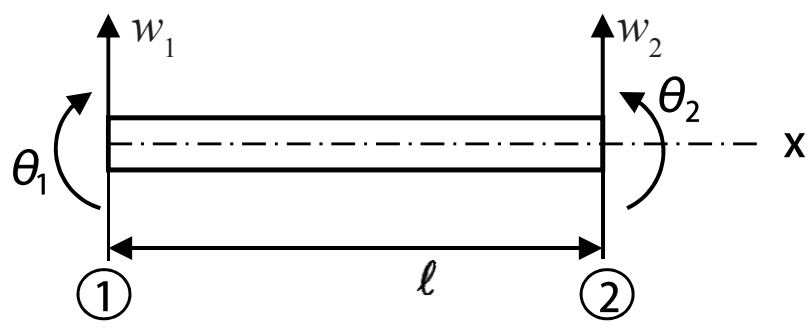

Fig. 2. Four degrees of freedom finite element model. 


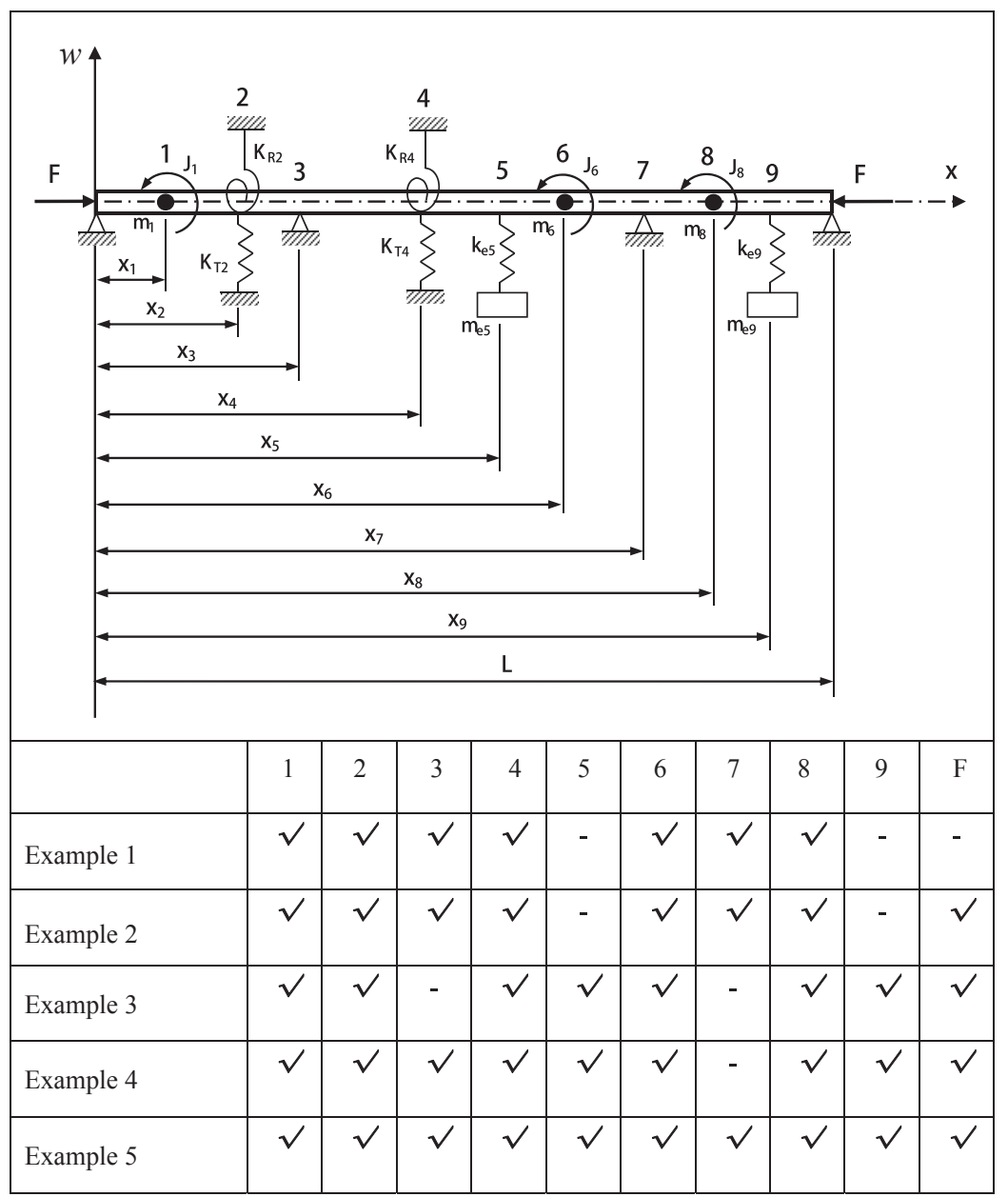

Fig. 3. A uniform pinned-pinned beam carrying three point masses, three rotary inertias, two linear springs, two rotational springs, two intermediate pinned supports and two mass spring sytems and subjected to axial load.

values obtained for the first five modes are presented and compared with the frequency values obtained for $F_{r}=0,0.25,0.5,0.75$ for compressive and tensile axial loads in Table 3.

It can be seen from Table 3 that when the number of spring-mass system increases the natural frequencies decrease for both load conditions. While the compressive axial force decreases the natural frequencies, the tensile force increases them. It can also be seen from Table 3 that for two spring-mass systems the percentage change in frequency values with the increasing $F_{r}$ is in the 6-17\% range while it is approximately $2 \%$ for the first mode. Therefore, it is more significant after the first mode. However, this significant percentage change in behaviour can be observed for all modes with no spring-mass and one spring-mass system. Also from the same table it is clear that; the third, fourth and fifth mode frequency values of one spring-mass system are almost the same as the second, third and fourth mode frequency values of no spring-mass system for all $F_{r}$ values. Besides, the fourth and fifth mode frequencies of two spring-mass systems are almost same as the third and fourth mode values of no spring-mass systems for all $F_{r}$ values. These similarities can be formulated as $\omega_{p+i}=\omega_{b i}(i=2,3, \ldots)$, where $\mathrm{p}$ denotes the total number of spring-mass systems attached and $\omega_{b i}$ denotes the $i$ 'th natural frequency of the beam carrying concentrated elements for no spring-mass systems, for the Bernoulli-Euler beam carrying concentrated elements such as point masses, rotary inertias, linear springs and rotational springs, subjected to compressive or tensile axial loads.

Furthermore, the first $(p+i)$ mode shapes of the beam carrying concentrated elements and $p$ spring-mass attachments have similar forms since the $(p+i)^{\prime}$ 'th mode frequency value of the beam with attachments is very close to the second mode frequency value of beam carrying only concentrated elements without any spring-mass attachment. 


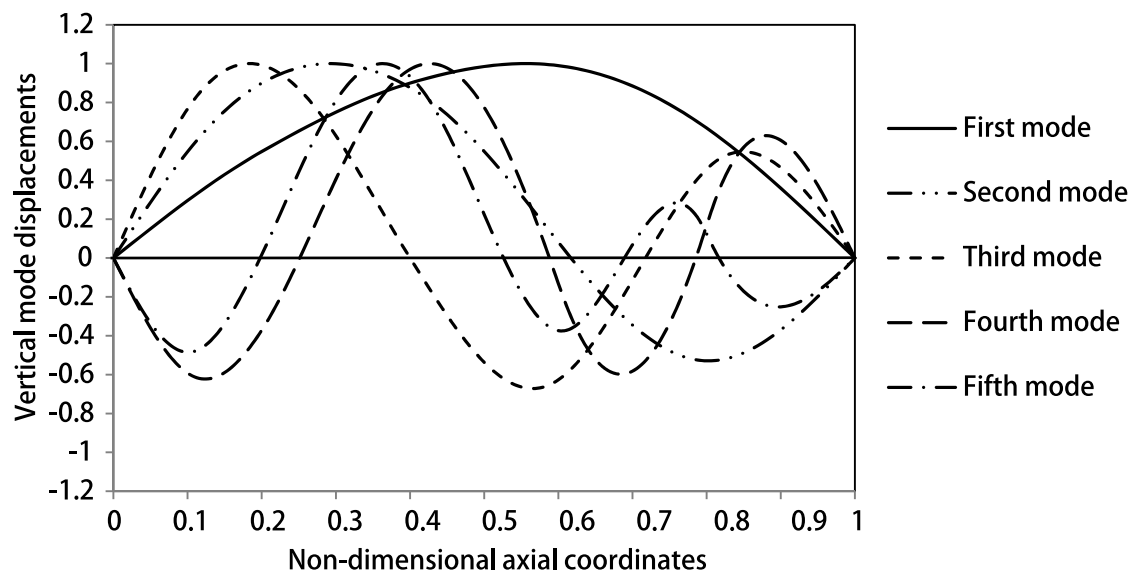

Fig. 4. The lowest five mode shapes of a single-span uniform beam carrying three point masses, two rotary inertias, two linear springs, two rotational springs and subjected to axial load.

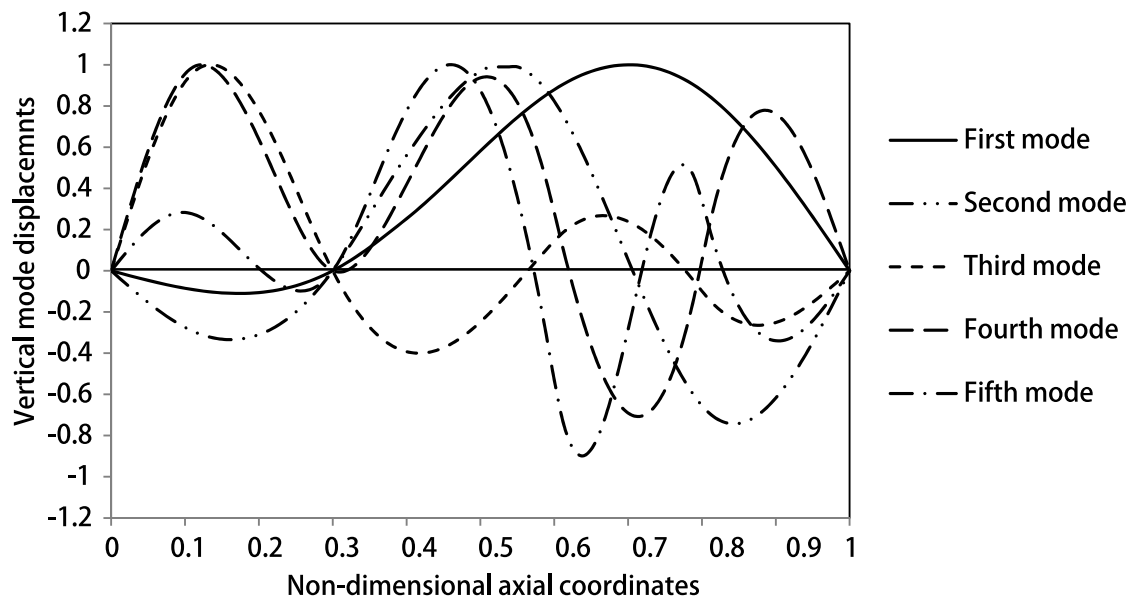

Fig. 5. The lowest five mode shapes of a two-span uniform beam carrying three point masses, two rotary inertias, two linear springs, two rotational springs and subjected to axial load.

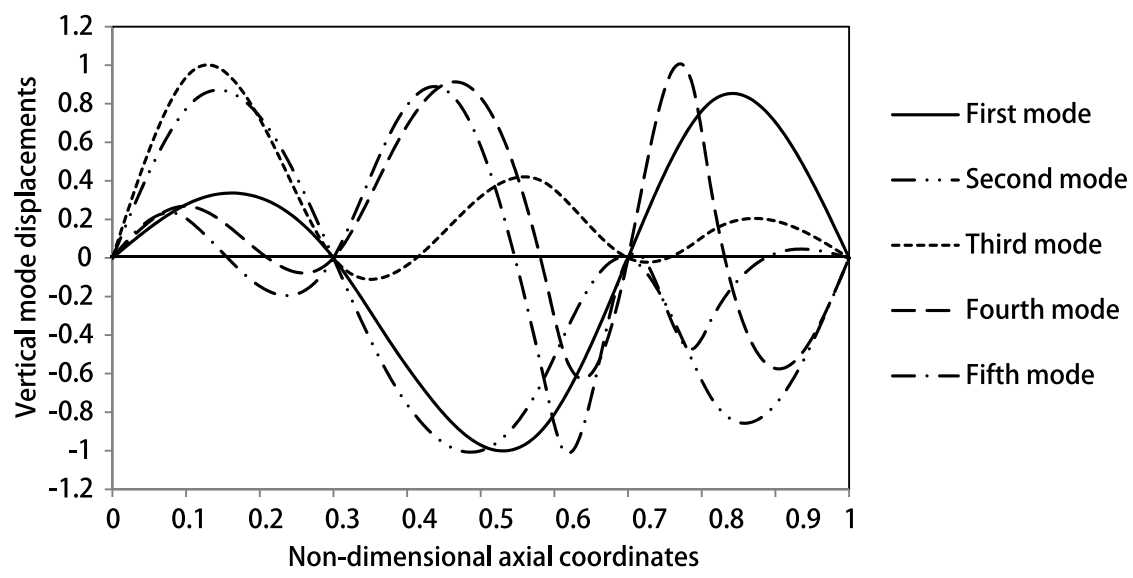

Fig. 6. The lowest five mode shapes of a three-span uniform beam carrying three point masses, two rotary inertias, two linear springs and two rotational springs and subjected to axial load. 


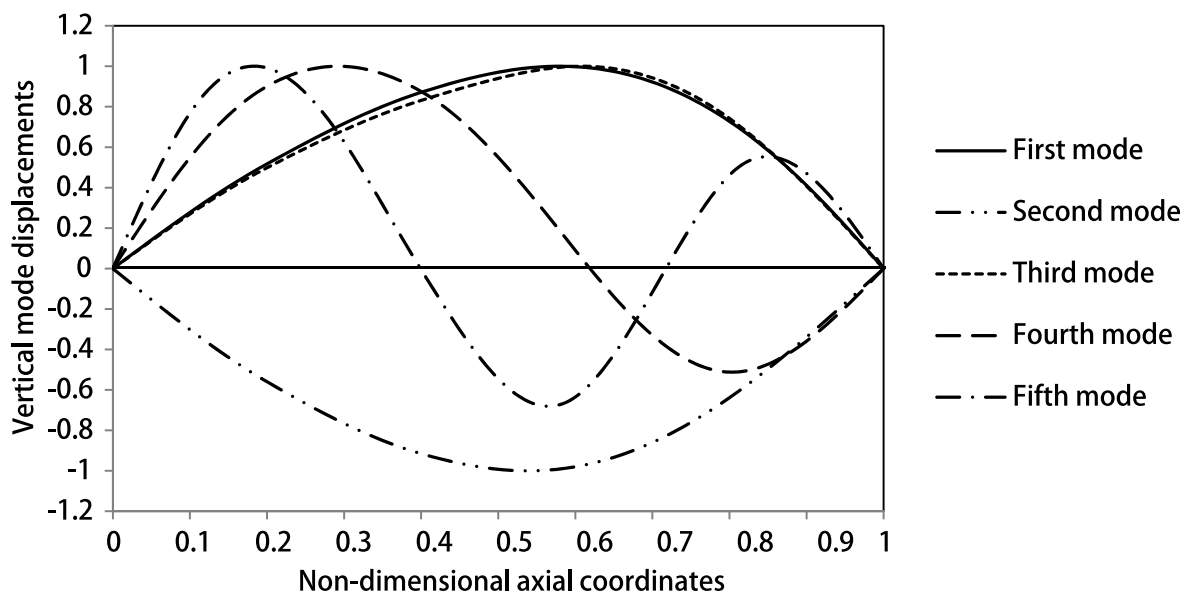

Fig. 7. The lowest five mode shapes of a single-span uniform beam carrying three point masses, two rotary inertias, two linear springs, two rotational springs and two spring-mass systems and subjected to axial load.

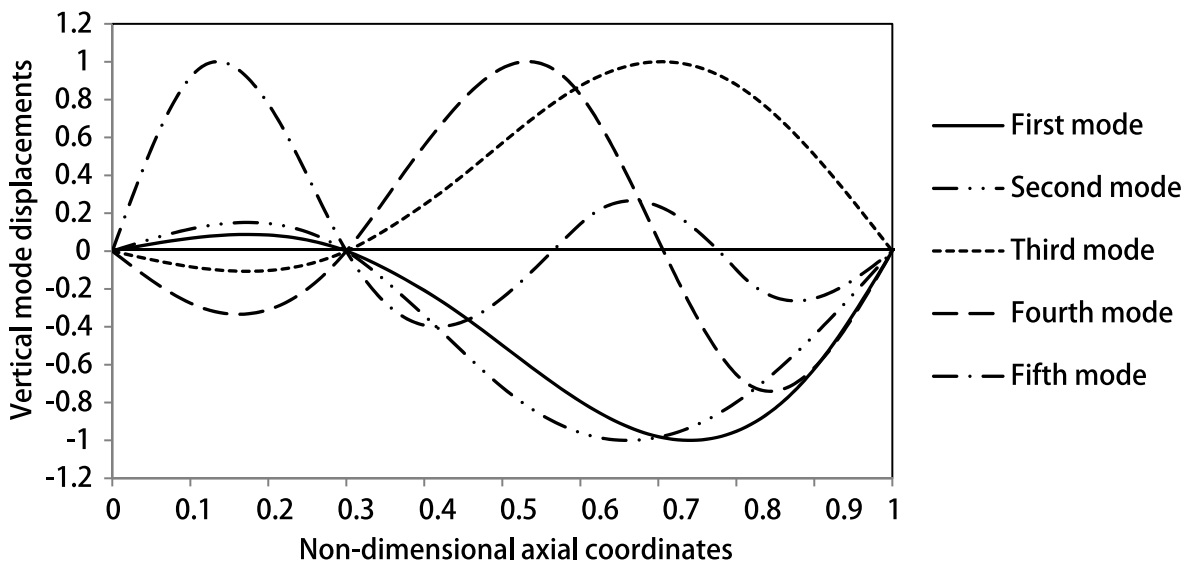

Fig. 8. The lowest five mode shapes of a two-span uniform beam carrying three point masses, two rotary inertias, two linear springs, two rotational springs and two spring-mass systems and subjected to axial load.

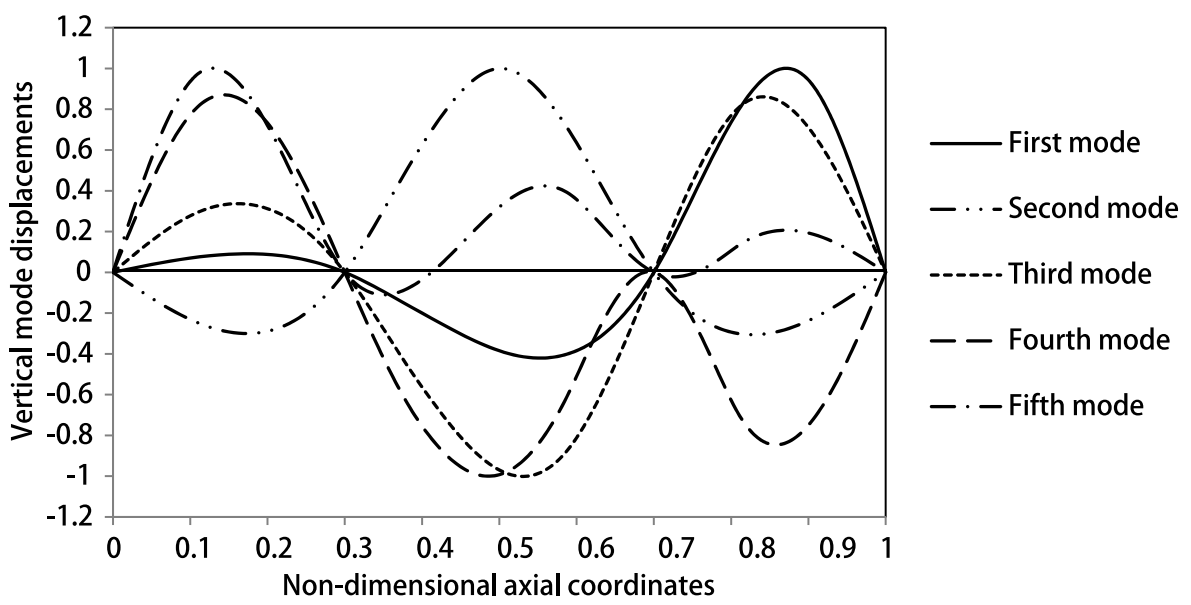

Fig. 9. The lowest five mode shapes of a three-span uniform beam carrying three point masses, two rotary inertias, two linear springs, two rotational springs and two mass spring sytems and subjected to axial load. 
These results are in harmony with those of Lin and Tsai, [10] and Yesice and Demirdag, [18] except for the fact that in Lin and Tsai, [10] the above relation is given only for the Bernoulli Euler beam carrying multi spring-mass systems but no axial load or concentrated elements while in Yesilce and Demirdag, [18] it is only given for the Timoshenko beams carrying multi spring-mass systems subjected to axial load but no concentrated elements.

Figure 7 shows the lowest five mode shapes of a single-span uniform beam carrying three point masses, two rotary inertias, two linear springs, two rotational springs and two spring-mass systems and subjected to axial load.

In the fourth example (see Fig. 3) the uniform two-span Bernoulli-Euler beam carrying concentrated elements and subjected to axial load are considered. In the fourth example one pinned support $\left(\xi_{3}=0.3\right)$ is added to the beam while the remaining data of this example are same of example 3. Corresponding Table 4 shows similar results obtained in Table 3. However, the frequency values at this point decreases with the increasing number of springmass-systems. Also, this decrease in ratio is highest in the three-span beam. From the Table 4 one can see the same relation of $\omega_{p+i}=\omega_{b i}(i=2,3, \ldots)$ for the Bernoulli-Euler beam carrying concentrated elements such as point masses, rotary inertias, linear springs and rotational springs subjected to compressive or tensile axial loads.

Figure 8 shows the lowest five mode shapes of a two-span uniform beam carrying three point masses, two rotary inertias, two linear springs, two rotational springs and two spring-mass systems and subjected to axial load.

In the fifth example (see Fig. 3) the uniform three-span Bernoulli-Euler beam carrying concentrated elements and subjected to axial load are considered. In the fifth example two pinned supports $\left(\xi_{3}=0.3, \xi_{7}=0.7\right)$ are added to the beam while the remaining data of these examples are the same as in example 3. Corresponding Table 5 shows similar results obtained in Table 4.

Figure 9 shows the lowest five mode shapes of a three-span uniform beam carrying three point masses, two rotary inertias, two linear springs, two rotational springs and two spring-mass systems and subjected to axial load.

Consequently, the first $(p+i)$ mode shapes of the beam carrying concentrated elements and $\mathrm{p}$ spring-mass attachments have similar forms since the $(p+i)$ 'th mode frequency value of the beam with attachments is very close to the second mode frequency value of the beam carrying only concentrated elements without a spring-mass attachment.

\section{Conclusions}

In this paper, free vibration analysis of beams carrying a number of various concentrated elements subjected to the axial load is performed using a Bernoulli-Euler beam assumption and FEM. The obtained results of the presented examples provide good agreement with available results in the literature. Especially $\left(\omega_{p+i}=\omega_{b i}\right)$ relation given by Lin and Tsai, [10] and Yesilce and Demirdag, [18] is clearly verified with our approach. Therefore, our results may construct a valid foundation for checking the accuracy and reliability of different methods as not many studies have been carried out in this area. Therefore, the results presented in this paper will be significant in this aspect.

\section{Nomenclature}

A Cross-sectional area of the beam

E Modulus of elasticity

I Secod moment of area of cross section

$L \quad$ Length of beam

$\{q\} \quad$ Nodal coordinate vector

$\{\dot{q}\} \quad$ Velocity vector

$T \quad$ Kinetic energy

$U \quad$ Strain energy

$V \quad$ Work done by an axial force

$\ell \quad$ Elemental length of beam

$w$ Nodal deflection

$\theta \quad$ Bending slope

$x$ Coordinate along the axis of the beam

$F \quad$ Axial force (Compression or tensile) 


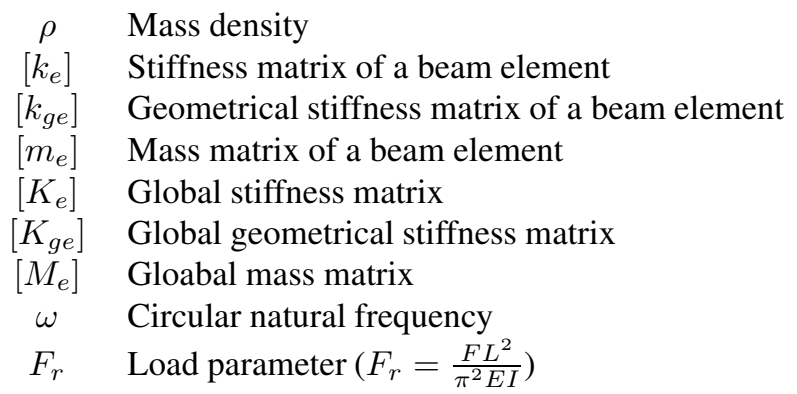

\section{References}

[1] R.D. Blevins, Formulas for natural frequencies and mode shapes, New York: Van Nostrand Reinhold, (1979).

[2] W. Weaver, S.P. Timoshenko and D.H. Young, Vibration problems in engineering, fifth edition, D.Van Nostrand Company, New York, (1990).

[3] F. Borbon and D. Ambrosini, On free vibration analysis of thin-walled beams axially loaded, Thin-Walled Structures 48 (2010), $915-920$.

[4] D.W. Chen and J.S. Wu, The exact solutions for the natural frequencies and mode shapes of non-uniform beams with multiple spring-mass systems, Journal of Sound and Vibration 255 (2002), 299-322.

[5] D.W. Chen, The exact solutions for the natural frequencies and mode shapes of non-uniform beams carrying multiple various concentrated elements, Structural Engineering and Mechanics 16 (2) (2003), 153-176.

[6] H.Y. Lin and Y.C. Tsai, On the natural frequencies and mode shapes of a uniform multi-span beam carrying multiple point masses, Structural Engineering and Mechanics 21 (3) (2005), 351-367.

[7] M. Gürgöze, On the alternative formulations of the frequency equation of a Bernoulli Euler beam to which several spring-mass systems are attached in span, Journal of Sound and Vibration 217 (1998), 585-595.

[8] S.M. Hashemi and M.J. Richard, Free vibration analysis of axially loaded bending torsion coupled beams: A dynamic finite element, Computers and Structures 77 (2000), 711-724.

[9] H.Y. Lin and Y.C Tsai, On the natural frequencies and mode shapes of a multi-step beam carrying a number of intermediate lumped masses and rotary inertias, Structural Engineering and Mechanics 22 (6) (2006), 701-717.

[10] H.Y. Lin and Y.C. Tsai, Free vibration analysis of a uniform multi-span beam carrying multiple spring-mass systems, Journal of Sound and Vibration 302 (2007), 442-456.

[11] H.Y. Lin, Dynamic analysis of a multi-span uniform beam carrying a number of various concenrated elements, Journal of Sound and Vibration 309 (2008), 262-275.

[12] H.Y. Lin, On the natural frequencies and mode shapes of a multi span Timoshenko beam carrying a number of various concentrated elements, Journal of Sound and Vibration 319 (2009), 593-605.

[13] S. Naguleswaran, Transverse vibrations of an Euler-Bernoulli uniform beam carrying several particles, International Journal of Mechanical Sciences 44 (2002), 2463-2478.

[14] S. Naguleswaran, Transverse vibration of an Euler-Bernoulli uniform beamon up to five resilient supports including ends, Journal of Sound and Vibration 261 (2003), 372-384.

[15] J.R. Wang, T.L. Liu and D.W. Chen, Free vibration analysis of a Timoshenko beam carrying multiple spring-mass systems with the effects of shear deformation and rotary inertia, Structural Engineering and Mechanics 26 (2007), 557-564.

[16] J.S. Wu and H.M. Chou, A new approach for determining the natural frequencies and mode shapes of a uniform beam carrying any number of sprung mass, Journal of Sound and Vibration 220 (1999), 451-468.

[17] J.S. Wu and D.W. Chen, Free vibration analysis of a Timoshenko beam carrying multiple spring-mass systems by using the numerical assembly technique, International Journal for Numerical Methods in Engineering 50 (2001), 1039-1058.

[18] Y. Yesilce and O. Demirdag, Effect of axial force on free vibration of Timeshenko multi-span beam carrying multiple spring-mass systems, International Journal of Mechanical Sciences 50 (2008), 995-1003. 

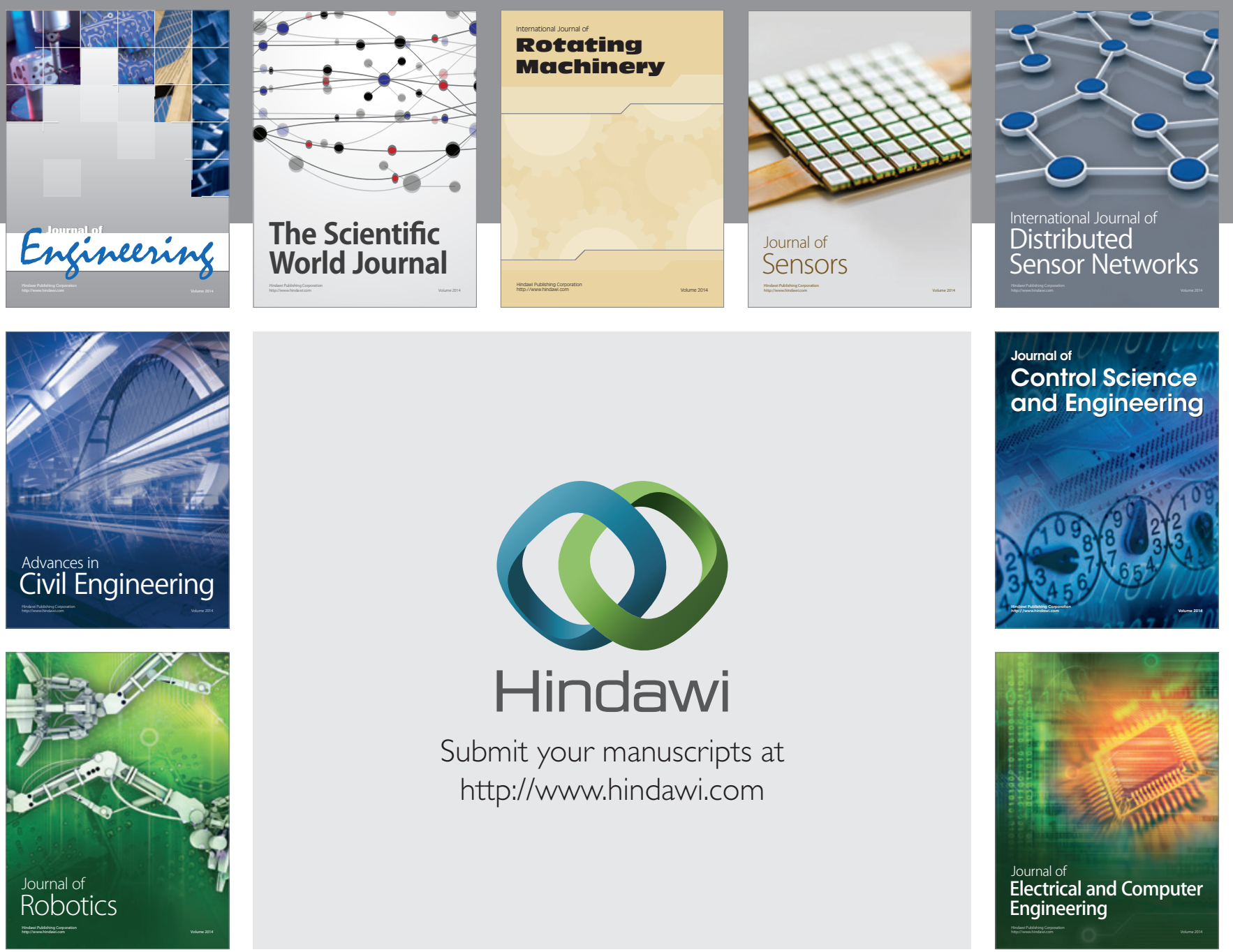

Submit your manuscripts at

http://www.hindawi.com
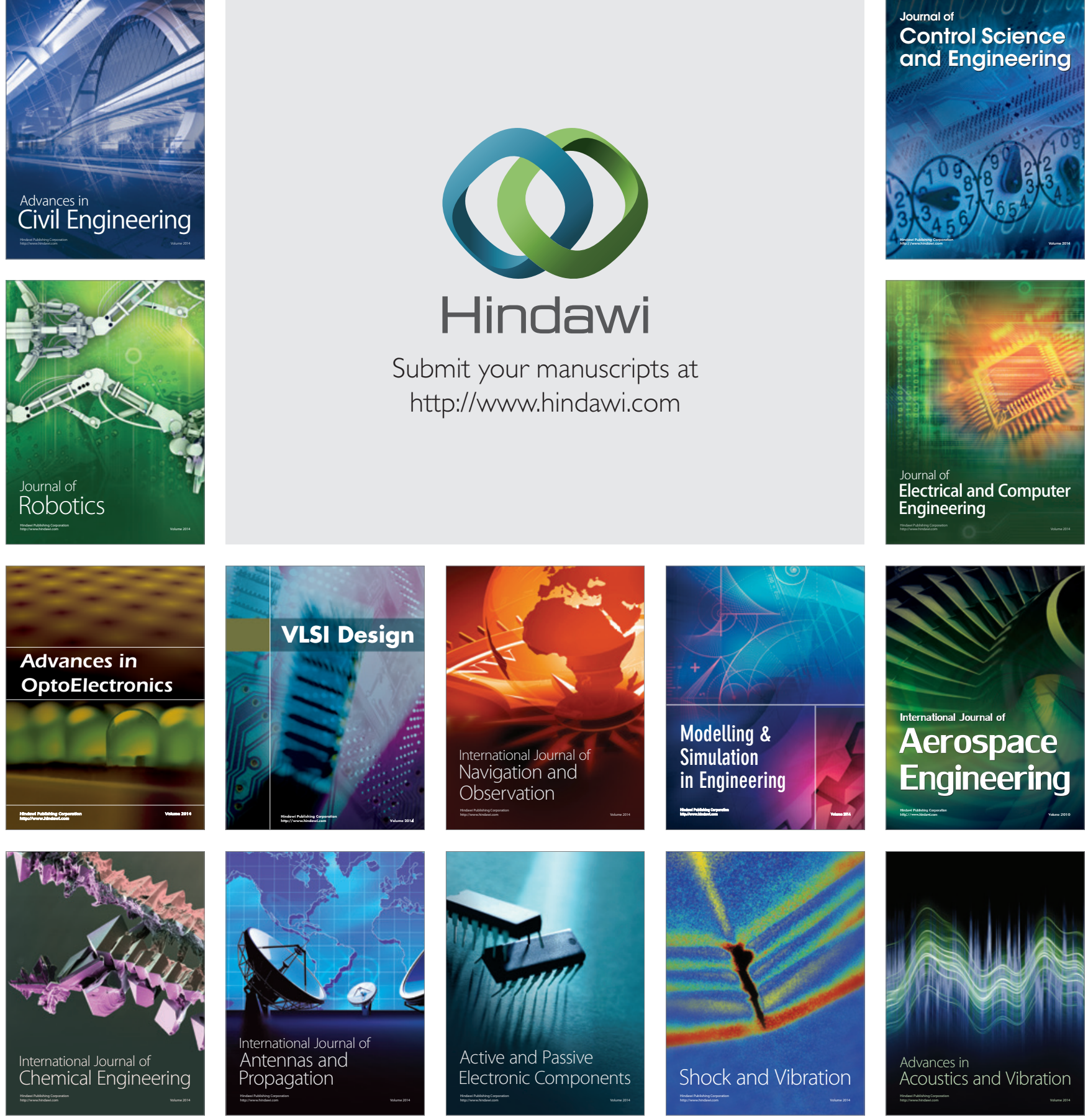